\title{
Longitudinal changes in QT interval variability and rate adaptation in pregnancies with normal and abnormal uterine perfusion
}

\begin{abstract}
Mathias Baumert ${ }^{1}$, Andrea Seeck ${ }^{2}$, Renaldo Faber ${ }^{3}$, Eugene Nalivaiko ${ }^{4}$ and Andreas Voss ${ }^{2}$
The maternal heart significantly adapts to the circulatory needs of pregnancy, but the effect of pregnancy on ventricular repolarization is poorly understood. The aim of this study was to quantify longitudinal changes in ventricular repolarization during pregnancy. Monthly electrocardiographs (ECGs) were recorded in 32 pregnant women with normal uterine perfusion and 32 pregnant women with abnormal perfusion, starting from the 20th week of gestation until 3 days postpartum. Ventricular repolarization was assessed through various QT interval variability and heart rate adaptation measures. The pregnancy outcomes of all women with normal perfusion were normal. Among pregnancies with abnormal uterine perfusion, 15 pregnancy outcomes were normal, but 17 pregnancies developed preeclampsia and/or small-for-gestational-age infants. In pregnancies with normal perfusion, the $\mathrm{QT}_{\mathrm{c}}$ interval was unaltered, but the QT interval-heart rate hysteresis lag was shorter and the QT interval-heart rate regression residual was higher compared with those of a control group of 10 healthy non-pregnant women. Pregnancies with abnormal uterine perfusion that developed pathological outcomes showed significantly smaller QT interval-heart rate regression residuals and a trend towards shorter $\mathrm{QT}_{\mathrm{c}}$ intervals compared with pregnant women with normal perfusion. In conclusion, pregnancy has a significant effect on ventricular repolarization. Pregnancies with abnormal uterine perfusion and subsequent pathological outcomes are paralleled by changes in ventricular repolarization that precede clinical symptoms.
\end{abstract}

Hypertension Research (2010) 33, 555-560; doi:10.1038/hr.2010.30; published online 12 March 2010

Keywords: preeclampsia; pregnancy; QT interval; QT variability; uterine perfusion

\section{INTRODUCTION}

Pregnancy leads to hemodynamic changes, which include the decrease in mean arterial pressure, peripheral vascular resistance as well as the increase in circulating volume, heart rate and cardiac output. Pregnancy has also been associated with left ventricular remodeling that results from the increased work load. ${ }^{1}$ In preeclamptic pregnancy, which is characterized by hypertension and proteinuria, the hypertrophy is even more pronounced. An animal study suggests that the increased ventricular myocyte volume observed during pregnancy is due to cell elongation, which was found to be more pronounced in preeclampsia. ${ }^{2}$

Apart from those structural changes, little is known about electrophysiological cardiac alterations during pregnancy. However, there is evidence suggesting that pregnancy can exacerbate preexisting underlying arrhythmias or even cause de novo arrhythmias. ${ }^{3-5}$ Multiple factors might be involved in the arrhythmogenesis, including increased hemodynamic stress on the cardiovascular system. Ventricular repolarization might be altered during pregnancy because of changes in circulating hormones, electrolyte levels ${ }^{6}$ and increased sympathetic neural outflow, ${ }^{7}$ thereby inducing ventricular arrhythmias. Ventricular repolarization dynamics can be assessed globally through QT interval (variability) in the body surface electrocardiograph (ECG).

Previous studies on ECG changes during pregnancy have been mainly focused on heart rate variability (beat-to-beat RR interval variability). ${ }^{8}$ Respiratory sinus arrhythmia was shown to have reduced, ${ }^{9,10}$ suggesting that the vagal neural outflow to the sinoatrial node of the heart is decreased during pregnancy and possibly caused by the reduction in tidal volume.

The aim of this study was to investigate variability and heart rate adaptations of the QT interval for pregnancies with and without abnormal uterine perfusion. It was hypothesized that abnormal perfusion of the uterine arteries and linked feedback mechanisms of the fetoplacentary unit are associated with increased stress on the maternal heart which in turn affect maternal ventricular repolarization.

\section{METHODS}

\section{Subjects}

In this longitudinal study, 32 healthy pregnant women with normal uterine perfusion (CON, age: $28 \pm 4$ years) and 32 women with abnormal uterine perfusion (AP, age: $28 \pm 6$ years) were enrolled. Maternal age, gravidity and

\footnotetext{
${ }^{1}$ School of Paediatrics and Reproductive Health and School of Medicine, University of Adelaide, Adelaide, Australia; ${ }^{2}$ Department of Medical Engineering and Biotechnology, University of Applied Sciences Jena, Germany; ${ }^{3}$ Department of Obstetrics, University of Leipzig, Leipzig, Germany and ${ }^{4}$ School of Biomedical Sciences, University of Newcastle, Newcastle, Australia

Correspondence: Dr M Baumert, School of Paediatrics and Reproductive Health, The University of Adelaide, 55 King William Road, Adelaide 5005, Australia. E-mail: mathias.baumert@adelaide.edu.au
}

Received 3 August 2009; revised and accepted 1 February 2010; published online 12 March 2010 
parity were comparable between both groups. Furthermore, all pregnancies were singleton and without clinical signs of cervical incompetence. Perfusion of the uterine arteries was assessed in the 20th week of gestation using a LOGIQ 9 ultrasound machine (GE, Solingen, Germany) with a $5 \mathrm{MHz}$ convex transducer. Uterine perfusion was defined as abnormal if bilateral notching was present and/or the mean pulsatility index (the maximum vertical excursion of the blood velocity waveform divided by its mean height) of both uterine arteries was greater than $1.45 .{ }^{11}$ In women with abnormal uterine perfusion, the Doppler measurement was validated in the 24th week of gestation. In addition, a control group of 10 healthy non-pregnant women aged $27 \pm 3$ years was recruited.

The following pathological pregnancy outcomes were considered in this study: (i) hypertension (a diastolic blood pressure reading $\geqslant 110 \mathrm{~mm} \mathrm{Hg}$ on any occasion or a reading of $\geqslant 90 \mathrm{~mm} \mathrm{Hg}$ on two consecutive diastolic blood pressure measurements that were at least $4 \mathrm{~h}$ apart); (ii) proteinuria ( $\geqslant 300 \mathrm{mg}$ total protein in a 24 -h urine collection or, if this was not available, positive proteinuria on two consecutive occasions at least $4 \mathrm{~h}$ apart); (iii) preeclampsia (hypertension plus proteinuria); and (iv) small-for-gestational-age infants (birth weight below the 10th percentile of an internal reference group).

The investigation conforms to the principles outlined in the Declaration of Helsinki. The University of Leipzig ethics committees' approval and written informed consent of all subjects have been provided.

\section{Data acquisition and preprocessing}

After the initial Doppler investigation in the 20th week of gestation, all women were monitored every 4 th week until 3 days postpartum. High-resolution ECG $(1600 \mathrm{~Hz}$, orthogonal three-lead system) was recorded over $30 \mathrm{~min}$ in the supine position under standardized resting conditions between 0800 and $1200 \mathrm{~h}$ as described before. ${ }^{12}$ For further processing we used the X-lead, in which the $\mathrm{T}$ wave was most pronounced.

\section{QT interval analysis}

To obtain beat-to-beat QT intervals, the algorithm proposed by Berger et al. ${ }^{13}$ was applied. Here, the operator defines a template QT interval by selecting the beginning of the QRS complex and the end of the T wave of one beat. The algorithm then finds the QT intervals of all other beats by determining how much each $\mathrm{T}$ wave must be stretched or compressed in time to best match the template. Thus, the QT interval measurement is relatively robust and insensitive to noise, as it does not rely exclusively on the exact determination of the $\mathrm{T}$ wave terminus. The following metrics were applied to quantify QT interval variability and rate dependence:

- QT: average QT interval without heart rate correction (in ms), quantifying the duration of ventricular repolarization globally;

- $Q T_{\text {var }}$ : variance of beat-to-beat QT intervals without heart rate correction (in $\mathrm{ms}^{2}$ ), quantifying overall variability in the QT interval;

- $Q T_{c}$ : average QT interval corrected for heart rate (in ms), estimating the QT interval at a projected RR interval of $1000 \mathrm{~ms}$. The method proposed by Malik et al. ${ }^{14,15}$ was applied. Briefly, a set of different regression models was employed to describe the relationship between QT interval and heart rate individually for each subject. The type of regression function (linear, hyperbolic, parabolic, exponential, logarithmic or shifted logarithmic) that fits the real data best and results in the smallest residual was then selected to compute $\mathrm{QT}_{\mathrm{c}}$;

- $Q T / H R$ regression residual: residual of the regression function that fits the QT interval/heart rate relationship best (in s), quantifying the strength of the QT interval dependence on heart rate and the goodness of the model, respectively;

- QT/HR regression exponent: regression exponent of the parabolic QT interval/heart rate model, describing how much the QT interval alters at a given change in heart rate; and

- QT/HR hysteresis lag: estimate of the hysteresis between heart rate changes and subsequent QT interval adaptation (in s), reflecting the time down to which previous heart rates have an effective influence on the current QT interval.

For further details on the computation of these measures see Malik et al., ${ }^{14}$ Pueyo et al. ${ }^{15}$

\section{Statistics}

For statistical analysis the monthly ECG measurements were binned according to the following classes:

- week 20: 18th-22nd week of gestation;

- week 24: 23th-26th week of gestation;

- week 28: 27th-30th week of gestation;

- week 32: 31st-34th week of gestation;

- week 36: 35th week of gestation until delivery; and

- pp day 3: 2nd-6th day postpartum.

One-way analysis of variance was conducted to test for time effects in pregnancies with normal perfusion. Post-hoc analyses were carried out based on Dunnett's multiple comparison tests with regard to the control group of non-pregnant women. Group differences between pregnancies with normal and abnormal uterine perfusion were investigated by applying two-way analysis of variance and Bonferoni's post-hoc comparisons. All measurements that were taken after the onset of clinical symptoms and during the subsequent pharmacological treatment were excluded from analysis.

\section{RESULTS}

All outcomes were normal in the group of pregnancies with normal uterine perfusion. In the group of pregnancies with abnormal uterine perfusion, 15 pregnancy outcomes were normal (AP-NO), 7 women delivered small-for-gestational-age infants without maternal hypertension and 10 women developed preeclampsia. For further analysis, we collated all cases of abnormal uterine perfusion and subsequent pathological outcomes (AP-PO). Abnormality of uterine perfusion was more pronounced in AP-PO compared with AP-NO (Table 1).

Longitudinal changes in heart rate, QT interval variability and rate adaptation in pregnancies with normal perfusion

Throughout the second half of gestation the average RR intervals of normal pregnant women were significantly shorter compared with those of non-pregnant women (Figure 1a). Post-hoc analysis revealed that the RR intervals remained significantly shortened 3 days postpartum $(P<0.0001$ for all post-hoc comparisons). Similarly, the average QT interval was shortened during the second half of pregnancy and remained shortened 3 days postpartum (Figure $1 \mathrm{~b}$; $P<0.001$ for all post-hoc comparisons). Using individual-specific regression models for heart rate correction, it was found that $\mathrm{QT}_{\mathrm{c}}$ intervals of pregnant women were not significantly different from those of non-pregnant controls (Figure 1c). Overall beat-to-beat QT interval variability $\left(\mathrm{QT}_{\mathrm{var}}\right)$ was significantly higher in pregnant women compared with non-pregnant women (Figure 1d; $P=0.02$ ). Post-hoc analysis revealed significantly increased $\mathrm{QT}_{\mathrm{var}}$ values during the 28th and 38th week of gestation $(P<0.05)$. The QT/HR hysteresis lag was significantly shortened during the second half of pregnancy (Figure 1e; $P=0.005)$.

The QT/HR regression exponent was not significantly different between pregnant and non-pregnant women (Figure 1f). On the contrary, the QT/HR regression residual was significantly higher in pregnant women $(P<0.0001)$ and did not reach normal levels 3 days postpartum (Figure 1g; $P<0.0001$ for all post-hoc comparisons).

\section{Heart rate, QT interval variability and rate adaptation in pregnancies with AP-NO respectively PO}

The average RR interval was significantly different between the three groups of pregnant women (Figure 1a; $P<0.0001$ ). Post-hoc analysis revealed the prolongation of RR intervals in AP-NO in comparison to those of CON during week $28 \quad(P<0.001)$, which subsequently returned to normal values. In $\mathrm{AP}-\mathrm{PO}$, the average $\mathrm{RR}$ interval was 
Table 1 Subject characteristics of pregnant women with normal uterine perfusion (CON), pregnant women with abnormal perfusion and normal outcome (AP-NO) and pregnant women with abnormal perfusion and pathological outcome (AP-PO)

\begin{tabular}{|c|c|c|c|}
\hline Parameter & $\operatorname{coN}(\mathrm{N}=32)$ & $A P-N O(\mathrm{~N}=15)$ & $A P-P O(\mathrm{~N}=17)$ \\
\hline Maternal age (years) & $28 \pm 4$ & $30 \pm 5$ & $26 \pm 6$ \\
\hline Obesity (\%) & 0 & $33^{a}$ & 12 \\
\hline Hypercoagulability (\%) & 0 & $3^{a}$ & 2 \\
\hline Initial systolic blood pressure (BP) (mm Hg) & $123 \pm 18$ & $129 \pm 16$ & $126 \pm 18$ \\
\hline Initial diastolic BP (mm Hg) & $72 \pm 9$ & $83 \pm 14^{a}$ & $80 \pm 12$ \\
\hline Initial measurement (weeks) & $20 \pm 1$ & $21 \pm 1$ & $20 \pm 1$ \\
\hline Primipara (\%) & 59 & 73 & 82 \\
\hline Mean uterine pulsatility index & $0.8 \pm 0.2$ & $1.5 \pm 0.2^{\mathrm{a}}$ & $1.9 \pm 0.4^{a, b}$ \\
\hline Rate of bilateral notch (\%) & 0 & $40^{\mathrm{a}}$ & $76^{a, b}$ \\
\hline Week of delivery & $39 \pm 1$ & $40 \pm 1$ & $35 \pm 3^{a, b}$ \\
\hline Birth weight (g) & $3450 \pm 427$ & $3359 \pm 433$ & $1970 \pm 583^{a, b}$ \\
\hline Rate of cesarean section (\%) & 28 & 20 & $82^{a, b}$ \\
\hline Rate of small-for-gestational-age infants (\%) & 0 & 0 & $65^{a, b}$ \\
\hline Hypertensive disorder (\%) & 0 & 0 & $59^{a, b}$ \\
\hline
\end{tabular}

Data presented as group means and s.d. or percentage, respectively.

asignificantly different compared to CON.

bSignificantly different compared to AP-NO.

also significantly longer during week 28 compared with CON $(P<0.001)$, but interestingly, remained prolonged during the subsequent measurement $(P<0.001)$.

The mean QT intervals were also significantly different between the three groups under investigation (Figure 1b). Post-hoc comparison showed prolonged QT intervals in AP-PO compared with CON during week $28(P<0.05)$ and significantly shortened QT intervals in AP-NO compared with CON during week $32(P<0.05)$.

Further, $\mathrm{QT}_{\mathrm{c}}$ was significantly different between the three groups (Figure 1c; $P=0.02$ ). Post-hoc analysis showed a trend for $\mathrm{QT}_{\mathrm{c}}$ interval shortening in AP-PO compared with CON during week 32 of pregnancy.

The QT/HR regression residual was significantly different between the three groups (Figure 1g; $P=0.0005$ ). Post-hoc analysis revealed significantly smaller QT/HR regression residuals in AP-PO compared with CON during the 28th week of gestation $(P<0.05)$.

Neither the QT/HR hysteresis lag, the QT/HR regression exponent, nor the $\mathrm{QT}_{\mathrm{var}}$ values were significantly different between pregnancies with normal and abnormal perfusion.

Correlations between heart rate, QT interval variability, rate adaptation and blood pressure

Although all measurements included in the analysis were taken under normotensive conditions, pregnancies with abnormal uterine perfusion had elevated systolic blood pressure levels compared with pregnancies with normal uterine perfusion (Figure $2 ; P<0.0001$ ). Post-hoc analysis showed increases in systolic blood pressure in AP-NO and AP-PO starting on the 28th week of gestation $(P<0.05)$ that remained elevated for at least 3 days postpartum. Similarly, diastolic blood pressure levels were also significantly elevated in pregnancies with abnormal perfusion compared with pregnancies with normal perfusion (Figure 2; $P<0.0001$ ). Post-hoc analysis showed significantly elevated diastolic blood pressure levels in AP-NO during weeks 20, 28, 32 and $38(P<0.05)$ and in AP-PO during week 20 and $28(P<0.001)$.

None of the investigated QT interval variability and rate adaptation measures was significantly correlated with systolic or diastolic blood pressure levels. On the contrary, the QT/HR regression residual $(r=-0.19, \quad P=0.002)$ and the QT/HR hysteresis lag $(r=0.25$, $P<0.001)$ were significantly correlated with the mean RR interval.

\section{DISCUSSION}

The major findings of this study are: (i) the rate-corrected $\mathrm{QT}_{\mathrm{c}}$ interval was not altered during normal pregnancy, but the QT/HR hysteresis lag was shorter and the QT/HR regression residual was higher compared with those of non-pregnant women; and (ii) pregnancies with abnormal uterine perfusion and subsequent pathological outcomes had significantly reduced QT/HR regression residuals and showed a trend towards shorter QTc intervals compared with pregnant women with normal perfusion.

\section{Heart rate, QT variability and rate adaptation in pregnancies} with normal perfusion

The heart rate acceleration (that is, RR interval shortening) that was observed during this study is a well-known phenomenon of pregnancy, yielding increased cardiac output and optimized oxygen and nutrients supply. This study further shows that the chronotropic effect of pregnancy persists for at least 3 days postpartum, indicating that heart rate normalization is associated with the normalization of circulating blood volume and peripheral resistance, outlasting the removal of the feto-placentary unit.

The increase in heart rate was paralleled by a significant shortening of the QT interval, reflecting the rate-dependence of the QT interval. When corrected for heart rate, the $\mathrm{QT}_{\mathrm{c}}$ interval of pregnant women was not significantly different from that of non-pregnant women, thus suggesting that the average duration of ventricular repolarization is not altered by pregnancy. In contrast, the dynamic process of QT interval adaptation to heart rate changes appears to be blunted during pregnancy, as indicated by higher QT/HR regression residuals. Correlation analysis indicates that QT/HR regression residuals increase at higher heart rates, that is the QT interval adaptability to heart rate weakens when the heart beats fast. At the same time the QT/HR hysteresis lag was found to have decreased during pregnancy, that is the time window of preceding RR intervals, which affect the current QT interval, shortened. This might be an indirect effect of the weaker QT rate-dependence at high heart rates.

QT interval adaptations to heart rate changes comprise of two processes: an initial rapid adjustment lasting a few seconds followed by a longer lasting adaptation taking several minutes. ${ }^{16}$ The latter was proposed to be modulated by catecholamines, where sympathetic 

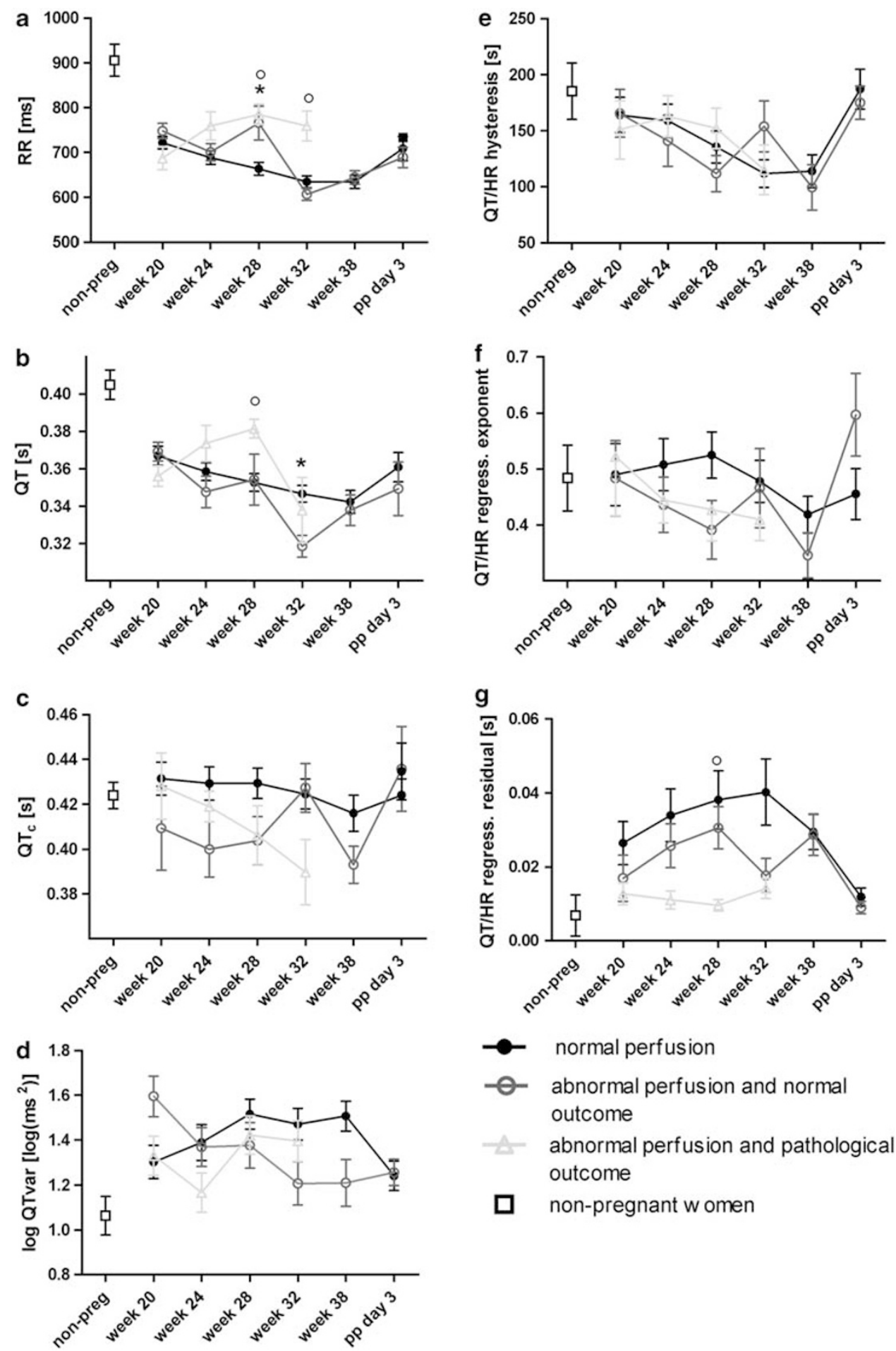

Figure 1 (a) Longitudinal changes in RR interval, (b) QT interval, (c) QT interval corrected for heart rate, (d) overall QT variability, (e) QT/RR hysteresis lag, (f) exponent of the QT/RR regression fit and (g) residual of the QT/RR regression fit during the second half of pregnancy and three days postpartum. The graphs show means and s.e.m. Circles indicate significant post-hoc test differences between abnormal uterine perfusion and subsequent pathological outcomes (AP-PO) and healthy pregnant women with normal uterine perfusion (CON). Asterisks indicate significant post-hoc test differences between AP-NO and CON.

activation results in an acceleration of the rate-adaptation through stimulation of $\mathrm{Na}^{+}-\mathrm{K}^{+}$pumps. ${ }^{17}$ The shortened QT/HR hysteresis lag observed during pregnancy might therefore be caused by increased sympathetic outflow to the ventricles that normalized within 3 days postpartum. In line with this argument, a rat model of pregnancy revealed that cardiac norepinephrine excretion increased with progressing gestation and returned to the normal levels after delivery. ${ }^{18}$ Human studies on sympathetic activity during pregnancy focused 


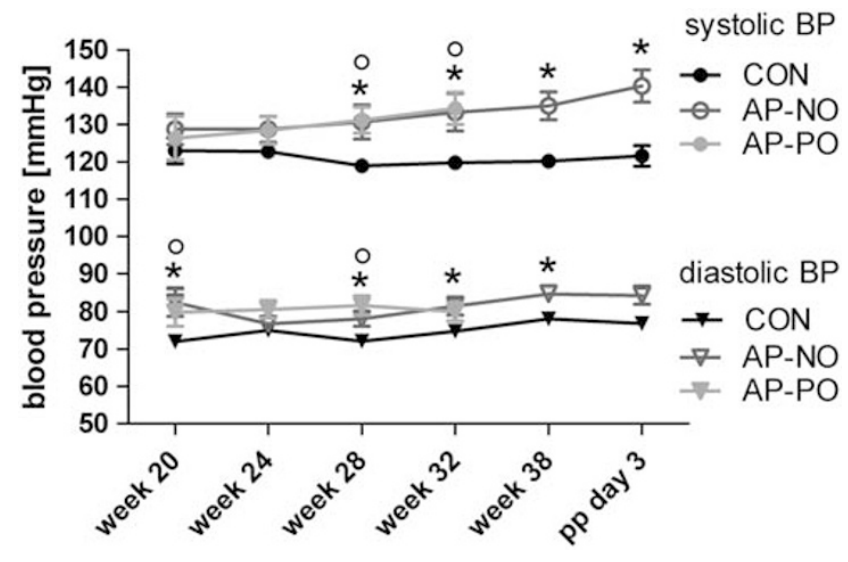

Figure 2 Longitudinal changes in systolic and diastolic blood pressure values during the second half of normal pregnancy and three days postpartum. CON, pregnancies with normal uterine perfusion and normal outcome; AP-NO, pregnancies with abnormal perfusion and normal outcome; and AP-PO, pregnancies with abnormal perfusion and pathological outcome. The graphs show means and s.e.m. Circles indicate significant post-hoc differences between AP-PO and CON. Asterisks indicate significant post-hoc differences between AP-NO and CON.

mainly on muscular sympathetic nerve activity and provided conflicting results, reporting increased ${ }^{7}$ and unaltered muscular sympathetic nerve activity levels. ${ }^{19}$ Taking further into consideration that the sympathetic nervous system is highly localized ${ }^{20}$ and the sympathetic neural outflow to muscles is not necessarily the same as that to the heart, it remains to be proven whether sympathetic cardiac activity is increased in normal human pregnancy and responsible for the observed changes in rate adaptation of the QT interval.

\section{Heart rate, QT interval variability and rate adaptation in pregnancies with abnormal uterine perfusion}

Abnormal uterine perfusion appears to have a significant effect on electrical cardiac activity. In pregnancies with abnormal uterine perfusion and subsequent pathological outcomes, in which the impairment of perfusion was more pronounced than in pregnancies with abnormal perfusion but normal outcome, heart rates were significantly slower compared with pregnancies with normal uterine perfusion. The lower heart rates appear counterintuitive, but might be caused by vagally driven baroreflex control of the elevated, yet normotensive blood pressure levels. The smaller QT/HR regression residuals observed in pregnancies with pathological outcomes might be an indirect effect of the slower heart rates (see above).

It is interesting to note that the rate corrected $\mathrm{QT}_{\mathrm{c}}$ interval of women with pathological pregnancy outcomes appears to shorten progressively throughout the second half of gestation, before the onset of clinical symptoms of hypertension or proteinuria. (The study design was such that measurements obtained after the clinical diagnosis were excluded.) The $\mathrm{QT}_{\mathrm{c}}$ shortening before pathological outcomes might be reflective of elevated sympathetic nerve activity that has been described in hypertensive pregnancy disorders. ${ }^{21}$ Based on muscular sympathetic nerve activity measurements it has been suggested that sympathetic overactivity precedes clinical symptoms of preeclampsia. $^{21}$

\section{Clinical implications}

This is the first study to show that normal pregnancy leads to subtle changes in the rate adaptation of ventricular repolarization. These alterations might contribute to the increased occurrence of arrhythmias that have been described previously. ${ }^{3-5}$ Severe abnormal uterine perfusion with subsequent pathological outcomes is associated with altered ventricular repolarization of the maternal heart that precedes clinical symptoms. Therefore, ECG assessment should be considered for pregnancy monitoring.

\section{Limitations}

All ECGs were recorded in the supine position. A more comprehensive understanding of maternal ventricular repolarization changes might be achieved through additional ECG assessments in the lateral position, in which significant hemodynamic and hormonal changes occur. ${ }^{22}$ Further, the narrow range of heart rates during rest might limit the precision of the QT/HR relationship estimation. Another limitation of this study is the collating of different pathological pregnancy outcomes in one group because of small sample sizes. Although the underlying pathologies are different, they both have in common abnormal uterine perfusion, which was more pronounced in pregnancies with pathological outcomes than in pregnancies with normal outcomes. The postpartum measurements were conducted between day 2 and 6 . Given the rapid changes that occur during the first week of the puerperium, this relatively wide time window might have introduced additional variance to the data.

\section{Conclusion}

Pregnancy has a significant effect on ventricular repolarization. Pregnancies with abnormal uterine perfusion and subsequent pathological outcomes show alterations in the QT interval and heart rate relationship that precede clinical symptoms. Further investigations are necessary to clarify which processes that are associated to abnormal uterine perfusion also effect cardiac electrophysiology.

\section{ACKNOWLEDGEMENTS}

This work has been supported by grants from the Deutsche Forschungsgemeinschaft (VO505/4-2, FA403-2). We are grateful to Dr Holger Stepan for recruiting patients.

1 Simmons LA, Gillin AG, Jeremy RW. Structural and functional changes in left ventricle during normotensive and preeclamptic pregnancy. Am J Physiol Heart Circ Physiol 2002; 283: H1627-H1633.

2 Bassien-Capsa V, Fouron JC, Comte B, Chorvatova A. Structural, functional and metabolic remodeling of rat left ventricular myocytes in normal and in sodiumsupplemented pregnancy. Cardiovasc Res 2006; 69: 423-431.

3 Gowda RM, Khan IA, Mehta NJ, Vasavada BC, Sacchi TJ. Cardiac arrhythmias in pregnancy: clinical and therapeutic considerations. Int J Cardiol 2003; 88: 129-133.

4 Nakagawa M, Katou S, Ichinose M, Nobe S, Yonemochi H, Miyakawa I, Saikawa T. Characteristics of new-onset ventricular arrhythmias in pregnancy. J Electrocardiol 2004; 37: 47-53.

5 Brodsky M, Doria R, Allen B, Sato D, Thomas G, Sada M. New-onset ventriculartachycardia during pregnancy. Am Heart J 1992; 123: 933-941.

6 Varon ME, Sherer DM, Abramowicz JS, Akiyama T. Maternal ventricular-tachycardia associated with hypomagnesemia. Am J Obstet Gynecol 1992; 167: 1352-1355.

7 Greenwood JP, Scott EM, Stoker JB, Walker JJ, Mary D. Sympathetic neural mechanisms in normal and hypertensive pregnancy in humans. Circulation 2001; 104: 2200-2204.

8 Malik M. Heart rate variability. Standards of measurement, physiological interpretation, and clinical use. Task force of the european society of cardiology and the north american society of pacing and electrophysiology. Eur Heart J 1996; 17: 354-381.

9 Faber R, Baumert M, Stepan H, Wessel N, Voss A, Walther T. Baroreflex sensitivity, heart rate, and blood pressure variability in hypertensive pregnancy disorders. J Hum Hypertens 2004; 18: 707-712.

10 Walther T, Wessel N, Baumert M, Stepan H, Voss A, Faber R. Longitudinal analysis of heart rate variability in chronic hypertensive pregnancy. Hypertens Res 2005; 28 : 113-118.

11 Papageorghiou AT, Yu CKH, Cicero S, Bower S, Nicolaides KH. Second-trimester uterine artery Doppler screening in unselected populations: a review. J Matern Fetal Neonatal Med 2002; 12: 78-88. 
12 Voss A, Malberg H, Schumann A, Wessel N, Walther T, Stepan H, Faber R. Baroreflex sensitivity, heart rate, and blood pressure variability in normal pregnancy. Am J Hypertens 2000; 13: 1218-1225.

13 Berger RD, Kasper EK, Baughman KL, Marban E, Calkins H, Tomaselli GF. Beat-to-beat QT interval variability-novel evidence for repolarization lability in ischemic and nonischemic dilated cardiomyopathy. Circulation 1997; 96: 1557-1565.

14 Malik M, Farbom P, Batchvarov V, Hnatkova K, Camm AJ. Relation between QT and RR intervals is highly individual among health subjects: implications for heart rate correction of the QT interval. Heart 2002; 87: 220-228.

15 Pueyo E, Smetana P, Caminal P, de Luna AB, Malik M, Laguna P. Characterization of QT interval adaptation to RR interval changes and its use as a risk-stratifier of arrhythmic mortality in amiodarone-treated survivors of acute myocardial infarction. IEEE Trans Biomed Eng 2004; 51: 1511-1520.

16 Franz MR, Swerdlow CD, Liem LB, Schaefer J. Cycle length dependence of human action-potential duration in vivo - effects of single extrastimuli, sudden sustained rate acceleration and deceleration, and different steady-state frequencies. J Clin Invest 1988; 82: 972-979.

17 Zaza A, Malfatto G, Schwartz PJ. Sympathetic modulation of the relation between ventricular repolarization and cycle length. Circ Res 1991; 68: 1191-1203.

18 Cohen WR, Galen LH, Vegarich M, Young JB. Cardiac sympathetic activity during rat pregnancy. Metab Clin Exp 1988; 37: 771-777.

19 Schobel HP, Fischer T, Heuszer K, Geiger H, Schmieder RE. Preeclampsia-a state of sympathetic overactivity. N Engl J Med 1996; 335: 1480-1485.

20 Esler M. Clinical application of noradrenaline spillover methodology: delineation of regional human sympathetic nervous responses. Pharmacol Toxicol 1993. 73. 243-253.

21 Fischer T, Schobel HP, Frank H, Andreae M, Schneider KTM, Heusser K. Pregnancyinduced sympathetic overactivity: a precursor of preeclampsia. Eur J Clin Invest 2004; 34: 443-448.

22 Almeida FA, Pavan MV, Rodrigues CIS. The haemodynamic, renal excretory and hormonal changes induced by resting in the left lateral position in normal pregnant women during late gestation. BJOG 2009; 116: 1749-1754. 